\title{
GRABGAM: A Gamma Analysis Code for Ultra-Low-Level HPGe SPECTRA
}

by

W. G. Winn

Westinghouse Savannah River Company

Savannah River Site

Aiken, South Carolina 29808

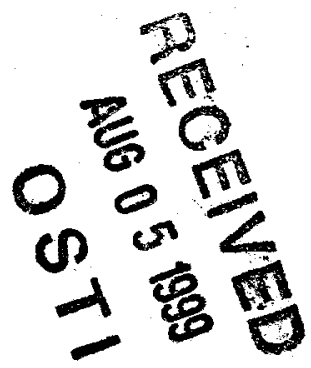

This paper was prepared in connection with work done under the above contract number with the U.S. Department of Energy. By acceptance of this paper, the publisher and/or recipient acknowledges the U.S. Government's right to retain a nonexclusive, royalty-free license in and to any copyright covering this paper, along with the right to reproduce and to authorize others to reproduce all or part of the copyrighted paper. 


\section{DISCLAIMER}

This report was prepared as an account of work sponsored by an agency of the United States Government. Neither the United States Government nor any agency thereof, nor any of their employees, makes any warranty, express or implied, or assumes any legal liability or responsibility for the accuracy, completeness, or usefulness of any information, apparatus, product, or process disclosed, or represents that its use would not infringe privately owned rights. Reference herein to any specific commercial product, process, or service by trade name, trademark, manufacturer, or otherwise does not necessarily constitute or imply its endorsement, recommendation, or favoring by the United States Government or any agency thereof. The views and opinions of authors expressed herein do not necessarily state or reflect those of the United States Government or any agency thereof.

This report has been reproduced directly from the best available copy.

Available to DOE and DOE contractors from the Office of Scientific and Technical Information, P. O. Box 62, Oak Ridge, TN 37831; prices available from (423) 576-8401.

Available to the public from the National Technical Information Service, U. S. Department of Commerce, 5285 Port Royal Road, Springfield, VA 22161. 


\section{DISCLAIMER}

Portions of this document may be illegible in electronic image products. Images are produced from the best available original document. 
GRABGAM: A GAMMA ANAIYSIS CODE FOR ULTRA-LOW-LEVEL HPGE SPECTRA

W.G. WINN

Westinghouse Savannah River Company, Aiken, SC 29808, USA

The GRABGAM code has been developed for analysis of ultra-lowlevel HPGe gamma spectra. The code employs three different size filters for the peak search, where the largest filter provides best sensitivity for identifying low-level peaks and the smallest filter has the best resolution for distinguishing peaks within a multiplet. GRABGAM basically generates an integral probability Ffunction for each singlet or multiplet peak analysis, bypassing the usual peak fitting analysis for a differential f-function probability model. Because $F$ is defined by the peak data, statistical limitations for peak fitting are avoided; however, the E-function does provide generic values for peak centroid, full width at half maximum, and tail that are consistent with a Gaussian formulism. GRABGAM has successfully analyzed over 10,000 customer samples, and it interfaces with a variety of supplementary codes for deriving detector efficiencies, backgrounds, and quality checks. 
GRABGAM: A GAMMA ANALYSIS CODE FOR ULTRA-LOW-IEVEL HPGE SPECTRA

W.G. WINN

Westinghouse Savannah River Company, Aiken, SC 29808, USA

\section{Introduction}

The GRABGAM code was developed for ultra-low level HPGe gamma spectrometry analysis at Savannah River Technology Center (SRTC), where it has continued to evolve since its 1985 inception [1]. The GRABGAM acronym is derived from Gamma-Ray Analysis BASIC Generated At MCA/PC. To address low-level śtatist̄ics, the codé utilïzes an integral probability formulism for deriving peak parameters. BY contrast, prior codes normally utilized differential Gaussian probability formulisms [2-3], and subsequent formulisms [4-14] also used this approach, although non-Gaussian methods have been applied by some [15-17]. GRABGAM peak detection is initiated by three filters, the widest giving the best sensitivity for identifying singlets and the narrowest serving to distinguish peaks within a multiplet. Final detection is characterized relative to both a determination level [18-19] and the traditional 95\% detection level recommended by currie [19]. GRABGAM also incorporates efficiency interpolations as a function of sample size, so that all of a limited size sample may be optimally counted without reduction or dilution to match a fixed geometry [20].

At SRTC, over 10,000 low-level samples have been successfully analyzed with GRABGAM. The bulk of these samples were counted by the HPGe detectors of the Ultra-Low-Level counting Facility [2122] and the Underground Counting Facility [18,23]. Typical samples were counted overnight or longer, which governed the overall throughput. Because of the very low concentrations of radionuclides in the samples, most of the spectra have included very few peaks, favoring singlet detection; however, various spectra, such as those from the 1986 Chernobyl accident [24-25], have included numerous peaks, providing ample testing of the multiplet analysis.

Throughout the evolution of GRABGAM, attention has been given to ease of use and helpful diagnostics. The code is operable from an IBM PC, and it provides a logical sequence of input frames for the user. The peak analysis printout provides typical peak data as well as isotope activity, measured and expected FWHM, and any nonsample peak backgrounds. A supplementary code allows the user to select weighted averages of isotope activities based on those of the individual peaks. Other support codes were also developed, and these address detector efficiency calibrations, backgrounds, isotope libraries, and diagnostics 


\section{GRABGAM Algorithms}

The major GRABGAM algorithms are the peak search, singlet analysis, multiplet analysis, minimum detectable amount (MDA) analysis, and library matching method. The algorithm discussion below denotes two different full widths at half maximum (EWHMs), namely a user-input energy-dependent FWHM* as well as the usual FWHM from the spectral peak analysis.

\subsection{Peak Search}

The peak search algorithms are described in Fig. 1, which displays three peak filters similar to second-derivatives utilized in other search algorithms [2-3] and initially developed by Mariscotti [26] for Gaussian peaks. The figure denotes that each filter "traps" or "ropes" an area $a_{f}$ for peak spectral counts, viz.

$a_{f}=g-b_{1}-b_{2} \pm\left(g+b_{1}+b_{2}\right)^{1 / 2}$

where $g$ is the number of counts in the $2 \mathrm{n}$ middle filter channels, $b_{1}$ and $b_{2}$ are the counts in adjacent $n$ filter channels, and the uncertainty is the $1-\sigma$ counting error of $a_{f}$. As each filter moves across the spectrum, it calculates test-peak areas $a_{f}$ based on the regions of interest (ROIs) of the filter. For each filter the background ROIs ( $b_{1}$ and $b_{2}$ counts) are each displaced $n$ channels from the gross peak ROI ( $g$ counts) to enhance the peak/noise ratio.

In GRABGAM a peak is found when the test $a_{f}$ exceeds its $1-\sigma$ counting error by a factor of at least 3. A peak location channel then approximates the centroid as the maximum $a_{f}$ in the peak vicinity. If two peaks are separated by $<0.5$ EWHM* they are combined as a slightly broadened single peak.

The three filters simultaneously trap their respective areas $a_{f}$ relative to the same spectral region, as shown in Fig. 1 . The smallest filter (2-pt rope) is first tested for the $a_{f}=3 \sigma$ criteria; if satisfied, this smallest filter subsequently finds the peak location to allow better resolution should the peak be within a multiplet. Should the smallest filter not indicate a peak, the middle (4-pt rope) is tested, and should it fail, the largest (8-pt rope) is tested. These progressively larger filters are more sensitive for trapping a detectable $a_{f}$ but provide less resolution for discriminating peaks within multiplets.

\subsection{Singlet Analysis}

The singlet analysis is activated when adjacent peaks are

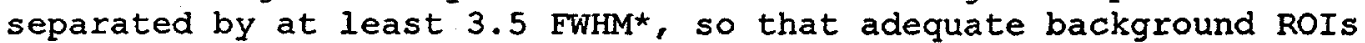
exist on both sides of the peak. The singlet analysis utilizes an integral peak analysis to accommodate low-level peaks more readily than the customary differential approach with peak fitting. In cases where the integral approach fails, other analysis options are applied as appropriate. 
The peak-adjacent background ROIs are found first. Both background ROIs have $m$ channels equaling the FWHM* of the peak location channel; however, the code limits the minimum background ROI to 3 channels. The ROI backgrounds $B_{1}$ and $B_{2}$ are initially placed adjacent to the peak location channel as shown in Fig. 2. Then first the $B_{1}$ ROI and then the $B_{2}$ ROI move down their respective sides of the peak while being tested for leveling out in appropriate background regions. As these ROIs move toward their destinations, their adequacy is tested against the criteria:

$$
\begin{aligned}
& B_{1 p}-B_{1} / m<2\left(B_{1 p}+B_{1} / m^{2}\right)^{1 / 2} \\
& B_{2 p}-B_{2} / m<2\left(B_{2 p}+B_{2} / m^{2}\right)^{1 / 2}
\end{aligned}
$$

where $B_{1 p}$ or $B_{2 p}$ denotes the counts in the channel just adjacent to the $B_{1} R O I$ or $B_{2} R O I$, on the peak side.

The peak gross region is determined from its adjacent background regions. As indicated by Eq. (2), the extreme end channels $h_{1}$ and $h_{2}$ of the peak gross region must have counts that are less than $2-\sigma$ above the adjacent background regions. If by chance the resulting gross region is less than 2 FWHM*, the average 3-point maximum channel $h_{\max }$ of the gross peak is determined and the gross region is defined by

$$
\begin{aligned}
\mathrm{h}_{1} & =\mathrm{h}_{\max }-\text { FWHM }^{*}-1 \\
\mathrm{~h}_{2} & =\mathrm{h}_{\max }+\text { FWHM }^{\star}
\end{aligned}
$$

Should the gross region be an uneven number of channels, it is forced to be even by adding a channel to the low side of the gross region, effectively lowering $h_{1}$ by 1 ; then the $B_{1}$ and $B_{2}$ regions are made adjacent to the final gross peak region. In every singlet peak analysis, the size of each background region is modified on its peak-remote side so that it has half the number of channels as the gross region. Using the ROI counts for gross counts $G$ and adjacent backgrounds $B_{1}$ and $B_{2}$, the peak acceptance must satisfy

$A=G-B_{1}-B_{2} \geq 3\left(G+B_{1}+B_{2}\right)^{1 / 2}$

where the peak area $A$ must be at least a factor of 3 greater than its counting error.

The centroid, FWHM, and tail are determined by utilizing the peak integral, subject to the following assumption. Typical HPGe spectral peaks exhibit a flat background $\left(B_{1}\right)$ on the lower-energy side of the peak that is somewhat higher than a similar one $\left(B_{2}\right)$ on the high-energy side of the peak. The additional background on the lower-energy side of the peak is due to the escape of gamma- 
induced electrons from the sensitive detector volume [2] and forward compton scattering within the sample, which are assumed to have distributions as shown in Fig. 3. The theoretical distribution (perfect resolution) shows a spike for the peak and a step function for the escape electrons plus forward compton events. The detected distribution illustrates the resolution effect. Fig. 3 denotes the parallels of the two distributions. In effect, if we view the peak with centroid $E_{g}$ as a differential probability function $f\left(E-E_{g}\right)$, the step background will be related to its integral probability. function $E\left(E-E_{g}\right)$, and we may write the differential gross gamma response $g(E)$ in the peak region as

$g(E)=A f\left(E-E_{g}\right)+b\left(1-F\left(E-E_{g}\right)\right)+c+v(E)$

where $A$ is the peak area, $b$ is the step background per keV, and $C+v(E)=z(E)$ is the background per keV upon which the peak distribution is superposed. For clarity, $z(E)$ is divided into its constant $c$ and energy-dependent $v(E)$ components. Integrating Eq. (5) over a single channel $j$ yields

$g_{j}=A\left(F_{j+1 / 2}-F_{j-1 / 2}\right)+b_{c}-b_{c}\left(F_{j+1 / 2}+F_{j-1 / 2}\right) / 2+c_{c}+v_{j}$

where $g_{j}$ denotes the counts in channel $j, F_{j+1 / 2}$ is the integral of $f\left(E-E_{g}\right)$ up to channel $j$ upper limit of $j+1 / 2, b_{c}$ and $c_{c}$ are the. constant background components per channel, and $v_{j}$ is $v(E)$ in channel units. The expression $\left(F_{j+1 / 2}+F_{j-1 / 2}\right) / 2$ is taken as the average $E\left(E-E_{g}\right)$ over channel $j . E q .(6)$ may be rearranged as a recursion relation to solve $F_{j+1 / 2}$ as

$F_{j+1 / 2}=\left[g_{j}-v_{j}+\left(A+b_{c} / 2\right) F_{j-1 / 2}-\left(b_{c}+c_{c}\right)\right] /\left[A-b_{c} / 2\right]$

which has an initial value $F_{i-1 / 2}=0$ associated with the lowest channel $i$ of the gross peak region. Actually GRABGAM uses a different but equivalent formalism for $F_{j+1 / 2}$, as developed by summing Eq. (6) from $j=i$ to $k$, viz

$$
\sum_{j=1}^{k}\left(g_{j}-v_{j}\right)=A\left(F_{k+1 / 2}-F_{i-1 / 2}\right)+(k-i+1)\left(b_{c}+C_{c}\right)-b_{c} \sum_{j=i}^{k}\left(F_{j+1 / 2}+F_{j-1 / 2}\right) / 2
$$

We note that the sum in the last term may be written as

$$
\sum_{j=1}^{k}\left(F_{j+1 / 2}+F_{j-1 / 2}\right) / 2=\left(F_{k+1 / 2}+F_{1-1 / 2}\right) / 2+\sum_{j=1}^{k-1} F_{j+1 / 2} .
$$


Recalling that $F_{i-1 / 2}=0$ and substituting Eq. (9) into Eq. (8) and rearranging yields

$F_{k+1 / 2}=\left[\sum_{j=i}^{k}\left(g_{j}-v_{j}\right)+b_{c} \sum_{j=i}^{k-1} F_{j+1 / 2}-(k-i+1)\left(b_{c}+c_{c}\right)\right] /\left[A-b_{c} / 2\right]$

With $F\left(E-E_{g}\right)$ solved per $F_{k+1 / 2}$, the following definitions result from the inverse function $E(E)$ for energy:

$$
\begin{aligned}
& \text { Cent }=E(0.50) \\
& \text { SigA }=E(0.16) \\
& \text { SigB }=E(0.84) \\
& \text { FWHM }=(2.355 / 2)(\text { SigA }- \text { SigB }) \\
& \text { Tail }=(\text { Cent-SigA } /(\text { SigB-Cent) }
\end{aligned}
$$

Effectively, this analysis provides operational definitions of the centroid, FWHM, and tail with analogy to a Gaussian peak. The FWHM definition is consistent with other methods for calculating Gaussian peak EWHMs, which show better than 108 agreement [27].

The current GRABGAM singlet analysis assumes $v_{j}=0$ for $\mathrm{Eq} \cdot(10)$, and thus singlet peaks on non-constant $z(E)$ backgrounds are not directly addressed. However, for a singlet such backgrounds have essentially no impact on the peak area calculation and only a minor impact on the peak centroid. On the other hand, multiplet peaks can be significantly impacted and a more realistic $v_{j}$ is modeled for this case.

There are cases where the F-integral analysis is not used, and these are flagged by negative values of the tail, as denoted in Fig. 3. For these cases the centroid is set as the middle channel of the gross region $\left(h_{1}+h_{2}\right) / 2$ and the FWHM is the gross width $\left(h_{2}-h_{1}+1\right)$, where again $h_{1}$ and $h_{2}$ are the end channels of the gross region. The negative tail and the abnormally large FWHM alert the user that the analysis may require further scrutiny.

\subsection{Multiplet Analysis}

A multiplet analysis is used for consecutive peaks that have their locations separated by less than $3.5 \mathrm{FWHM}^{\star}$. The FWHM* is assumed constant over the multiplet region and calculated based on the middle channel between the first and last peak of the multiplet. 
Initially background regions $B_{1}$ and $B_{2}$ above and below the multiplet are found similarly to that of a singlet peak, except that the $B_{1}$ region "ropes" down to a region on the low-energy side of the first multiplet peak and the $B_{2}$ region "ropes" down to a region on the high-energy side of the last multiplet peak, as shown in Fig 4. The $B_{1}$ and $B_{2}$ regions are required to be displaced at least a FWH* from their adjacent multiplet peaks. To give better representation against fluctuations, any points in the final BI or B2 regions that exceed their respective averages by $3 \sigma$ are removed from the average background per channel calculations.

The minima between adjacent peaks of the multiplet are found next. First, the gross minimum is sought; however, if the resulting minimum is not displaced at least one channel from both adjacent peak locations, a relative minimum is sought as the maximum difference between channel counts and the line connecting the adjacent peak maxima. Again, if this minimum is not displaced from both adjacent peak locations by at least one channel, then the minimum is taken as the inverse center of gravity of the adjacent peak maxima; this minimum is forced to be displaced at least one channel from its adjacent peaks. The latter two minimum searches provide better capability for defining a boundary between closely spaced multiplet peaks that display a shoulder rather than an obvious minimum, as illustrated in Fig. 5.

The total area $A_{M}$ of the multiplet is then calculated using the gross counts $G_{M}$ between the adjacent background regions as

$$
\begin{aligned}
& A_{M}=G_{M}-\left(N_{M} / 2 N_{1}\right) B_{1}-\left(N_{M} / 2 N_{2}\right) B_{2} \pm \sigma\left(A_{M}\right) \\
& \sigma\left(A_{M}\right)=\left[G_{M}+\left(N_{M} / 2 N_{1}\right)^{2} B_{1}+\left(N_{M} / 2 N_{2}\right)^{2} B_{2}\right]^{1 / 2}
\end{aligned}
$$

where $N_{M}, N_{1}$, and $N_{2}$ are the number of channels in the multiplet and background regions.

The integral F-function is generated in the same manner as that for the singlet analysis, as the sum of individual peak integrals within the multiplet yields the F-function for the multiplet. This is seen by noting how the individual peaks in the multiplet contribute as individual components of Eq. (5). In particular,

$g(E)=\sum_{m=1}^{n} A_{m} f\left(E-E_{m}\right)+\sum_{m=1}^{n} b_{m}\left(1-E\left(E-E_{m}\right)\right)+c+v(E)$

where $A_{m}$ and $E_{m}$ correspond to $A$ and $E_{g}$ of the $m^{\text {th }}$ of $n$ total multiplet peaks. Because the ratio of the step background $b_{m}$ and peak area $A_{m}$ should be essentially the same for each peak, we may substitute $b_{m}=\beta A_{m}$ in Eq. (13) yielding 
$g(E)=\sum_{m=1}^{n} A_{m} f\left(E-E_{m}\right)+\beta \sum_{m=1}^{n} A_{m}\left(1-E\left(E-E_{m}\right)\right)+c+v(E)$

which upon abbreviating $\sum_{m=1}^{n}$ as $\Sigma$ and rearranging yields

$$
g(E)=\sum A_{m} \frac{\sum A_{m} f\left(E-E_{m}\right)}{\sum A_{m}}+\beta \sum A_{m} \frac{\sum A_{m}\left(1-F\left(E-E_{m}\right)\right)}{\sum A_{m}}+c+v(E)
$$

where it is recognized that $f_{M}(E)=\sum A_{m} f\left(E-E_{m}\right) / \sum A_{m}$ is a differential probability function composed of peak-weighted probability functions of the multiplet, corresponding to integral probability $F_{M}(E)=\Sigma A_{m} E\left(E-E_{m}\right) / \Sigma A_{m}$. Noting that $A_{M}=\Sigma A_{m}$ is the total of peak areas of the multiplet, and also noting that $b_{M}=\beta \Sigma A_{m}=\Sigma b_{m}$ is the total of the step background components of the peaks, we may write

$g(E)=A_{M} f_{M}(E)+b_{M}\left(1-F_{M}(E)\right)+c+V(E)$

which is identical in form to Eq. (5) for a singlet peak. Thus, the peak multiplet integral $F_{M}(E)$ may be determined using the same algorithms developed for the singlet peak.

The multiplet analysis models $z(E)=c+v(E)$ as a cubic polynomial in E, so that the background under the multiplet is smoothly matched to its adjacent background regions [1]. The analysis also restricts the step backgrounds to realistic ranges, estimates peak centroids, and requires the total multiplet area $A_{M}$ to be consistent with its F-modeled background [1]. Unlike the singlet analysis, the individual peak areas $A_{m}$ can be strongly impacted by the underlying background.

As the $F$-function is generated, the fraction $F_{m / M}$ for each multiplet peak is determined by denoting the portion of $F$ between successive minima, whereby the end channels $h_{1}$ and $h_{2}$ of the gross multiplet region are also treated as minima. Referring to Fig. 4, the area $A_{m}$ of an individual multiplet peak is given by

$A_{m}=F_{m / M} A_{M} \pm\left(F_{m / M}\right)^{1 / 2} \sigma\left(A_{M}\right)$

The counting error for $A_{m}$ is approximated assuming that the $F_{m / M}$ fraction exactly represents the peak area; although this error is useful as a guide to the counting statistics, systematic errors 
due to peak overlap require correction. Such correction is provided by the PEAKPART code, which transforms the raw $A_{m}$ to corrected areas $\mathrm{X}_{\mathrm{m}}$ using the $\mathrm{n}$ linear equations

$A_{k}=\sum_{m=1}^{n} F_{k m} X_{m}$

where $F_{k m}$ is the fraction of the peak with area $X_{m}$ that lies in the ROI containing area $A_{k}$. The $F_{k m}$ are derived using the peak minima and maximum associated with each ROI assuming Gaussian distributions, and the transformation from $A_{m}$ to $x_{m}$ only redistributes the total multiplet area [1].

For multiplets with very low statistics, the method may encounter difficulties, and warning flags are issued to alert the user, as indicated in Fig. 4.

\subsection{MDA Analyses}

MDA analyses are performed for two types of peaks. In one case a statistically significant peak has been detected in the spectrum, but it has a background file peak that cancels the spectral peak. In the other case, the MDA flag of a gamma energy in the isotope library causes an analysis when no corresponding spectral peak is detected; here the FWHM* is used to define gross and background ROIs.

Both cases are calculated similarly. Each calculates a peak area $A$ and an error $\sigma(A)$, which essentially derives from an expression,

$A \pm \sigma(A)=[(A+B)-B] \pm(A+2 B)^{1 / 2}$

where $B$ is the effective background above which true signal A must be detected. Accordingly, from $A$ and $\sigma(A)$ the value of the background can be determined as

$B=\left(\sigma(A)^{2}-A\right) / 2$ 
Two types of MDAs are utilized in GRABGAM. One MDA is a determination level [19], which GRABGAM has defined so that results are presented as [18]

$\begin{array}{ll}A \pm \sigma(A) & \text { for } A \geq 3 \sigma(A) \\ <3 \sigma(A) ; x \text { for } A<3 \sigma(A)\end{array}$

where in the second case $x=A / \sigma(A)$ is provided, allowing conversion to $A \pm \sigma(A)$ if desired. The other MDA result uses the Currie definition of detection limit [19], which is given as

$<2.71+4.65 B^{1 / 2}$

and by itself is insufficient for conversion to $A \pm \sigma(A)$. Overall, the calculation of both MDAs provides the user more options for interpretation.

\subsection{Library Matching Method}

The peak data are merged with matching library data as the code executes. Each peak is analyzed in monotonically increasing order with energy. After each peak centroid and energy is determined, the monotonically ordered energies of the isotope library are stepped up to the region of the centroid energy to seek a match. If a match is found, the isotope data associated with the energy is transferred as part of the peak data for later calculations. If no match is found, the peak is labeled as "no-1ib" and a set of default parameters (1008 branching, zero decay time, etc) is assigned. This essentially one-dimensional matching approach speeds the search relative to a two-dimensional lookup technique.

For library flagged MDAs that do not correspond to detected peaks, the code calculates these cases and simultaneously merges them within the above sequence in order of gamma energy. In this process the energies/MDA flags of the entire isotope library are stepped monotonically between the peak energies found earlier, so that the new MDA analyses can be placed in the correct energy order within the peak list. 


\section{GRABGAM Operation}

GRABGAM is executed from any IBM compatible computer as a compiled BASIC program. The GRABGAM operational sequence is blocked out in Fig. 6, displaying the inputs, program, and outputs. Some of the output may be utilized by a set of supplementary analyses for further appraisal of the spectrum. The operational features [1] are summarized as follows.

\subsection{Input}

Before execution of GRABGAM, the user will have generated appropriate disk files for background peaks (backgnd.BKG), efficiency caibrations (DETIIBRY.IIB and/or detreff.DCF), isotope data (ISOIIBRY.LIB), and the spectrum itself (spectrum.CHN), as indicated in Fig. 6. The spectrum file is typically generated upon saving a counted spectrum using an EG\&G/ORTEC MCA/PC gamma spectroscopy system (ADCAM, MAESTRO, GAMMA VISION), although transformation of files from other MCA systems have also been achieved. The other files are generated from support codes accompanying the GRABGAM package [1].

During execution the user enters the following keyboard information: the type analysis (background subtract option and count-rate units); energy and FWHM* calibrations; efficiency selections (manual, file, and sample size); spectral analysis parameters (channel range, sample age, activity units, and energy limits for isotope peak identification).

\subsection{Program}

GRABGAM continues its execution following user keyboard inputs. The peak search algorithm sweeps through the spectrum initially, setting up candidates for the peak analysis algorithms which are then applied to singlets and multiplets. The peak analysis proceeds monotonically with the peak energies, which are simultaneously matched with library isotopes. Then the MDA analysis for flagged cases is executed and merged to the peak data list, after which the peak backgrounds are also merged. Final calculations are then applied to the peak data, including MDA. calculations for peaks that are not significant relative to their backgrounds.

Upon completion, the user is queried concerning the desire for supplementary analyses so that appropriate data files may generated. 


\section{3 output}

The user output is routed to the printer and CRT. Initially, the printer provides a comprehensive summary of the input data, as shown Fig. 7. Then, during the peak search, the CRT displays the candidate peaks as they are identified, as in Fig. 8. Finally, the peak results are printed in the format of Fig. 9.

Although the user output of Figs. 7-9 is fairly selfexplanatory, some clarifications are in order. In Fig. 7, the numerator and denominator factors respectively correspond to an efficiency normalization and the grams of sample. In Fig. 8 the peak area and its 8 -error correspond to the filter test area $a_{f}$. In Fig. 9 each peak has two energy values and two FWHM values. The upper values are determined by the peak analysis; the lower energy is the library matching energy, and the lower FWHM is FWHM*. Two values are also given for MDA cases: the upper value is for the determination level followed by a notation of $3 \sigma / x \sigma$, in reference to Eq. (21); the lower value is Currie detection limit followed by $M D A=c+b$ as a abbreviation of Eq. (22). Information on negative tails and multiplet notations are summarized in Figs. 3-4, but details are described elsewhere [1].

Supplementary analyses data output is routed to the printer and disk files. The printer produces a table of the multiplet data, as shown in Fig. 10, for use with the PEAKPART code. Five files (DATACALB.DAT, DATASPEC.DAT, DATAISOS.DAT, DATAPEAK.DAT, DATAMISC.DAT) are routed to disk for other supplementary analyses.

\subsection{Supplememtary Analyses}

Six supplementary analysis codes may be run following GRABGAM execution. A brief summary of each code is given below:

GRABISOS - groups and orders all peaks with regard to isotopic identification, allowing the user to select a weighted average of each isotopic activity for a more comprehensive analysis.

GRABBACK - produces peak background file backgnd.BKG based on the preceding GRABGAM spectral analysis for a detector background, as appropriate. It also has option to average with results from earlier peak background file results.

GRABCALB - produces detector efficiency file detreff.DCF based on preceding GRABGAM spectral analysis of calibration standard, as appropriate. It also provides for summing corrections using the spectrum peaks and continuum.

GRABDIAG - produces diagnostic table for studying presence of artifact peaks including Compton edges, backscatter, and escape peaks. 
GRABPLOT - provides interactive peak analysis on monitor screen for user selected peaks.

PEAKPART - refines the multiplet analysis to better correct for overlapping peak areas.

GRABISOS is the supplementary code used most frequently, as it is run as a routine part of each sample analysis. Fig. 11 shows an example of the results printed by this code. A more detailed discussion of these codes is available [1], although beyond the scope of the present paper."

\section{Discussion}

\subsection{Philosophy of Ultra-Low-Level HPGe Spectral Analysis}

Spectral analyses of low-level gamma peaks are always subject to statistical limitations with regard to peak fitting. Accordingly GRABGAM pursues an integral approach to peak analysis. Theoretically, the GRABGAM peak integral function $F$ is automatically corrected for its background, regardless of the actual peak shape. Overall, the F-function defines a consistent centroid, FWHM, and tail for a singlet peak, with no requirement on the detector performance for producing ideal peaks. Of course, even the integral approach may fail due to statistical limitations, and when this happens a basic ROI analysis provides an estimate so that the peak can be considered.

Singlets are anticipated to be predominant in low-level gamma spectrometry. Due to resolution and statistical limits, the GRABGAM peak search routine does not distinguish peaks that are separated by less than 0.5 FWHM*; however, the F-analysis for the FWHM and tail of the resulting singlet peak signals that the peak may be a composite. The multiplet analysis is similarly impacted, as a peak within a multiplet may also be a composite. Overall, GRABGAM experience in counting low-level samples has not been hampered by such hidden composite peaks, as the probability of such occurrences is generally low. Furthermore, often a composite peak can be resolved using peak ratios in conjunction with other gammas within the spectra.

GRABGAM calculates both a determination limit and a detection limit. The GRABGAM determination limit [18] of $A \geq 3 \sigma$ is comparable numerically to that of the Currie detection limit [19] 1958 probability detection), but both are always higher than the currie critical level [19] (58 false positive) for being detected above background. These three values can be derived as a function of background counts B, and are plotted in Fig. 12. GRABGAM assures that its minimum detection has a $1-\sigma$ statistical error no worse than 338 , while recorded detection at the Currie critical level has errors in excess of 608 . Instead of directly accepting peaks with such high errors, the GRABGAM philosophy is to flag MDA analyses for potentially useful peaks, and utilize the " $3 \sigma / x \sigma$ " formalism to extract the " $\sigma \sigma \pm \sigma$ " activity as appropriate. Consequently GRABGAM 
has a very low false positive acceptance in general, while providing an option for reconsidering the flagged MDA peaks.

Although the GRABGAM determination level and the Currie detection limit are comparable in magnitude, their associated MDAs have different interpretations. The Currie MDA is an anticipated detection level that is based on a priori reasoning. The GRABGAM MDA indicates that the detected peak was less than this amount, and thus uses a posteriori reasoning. Overall, the Currie MDA may be stated as the "minimum detectable amount" and the GRABGAM MDA be stated as the "minimum detected amount". An attractive feature of the GRABGAM MDA is that the detected values always exceed the MDA, whereas the Currie MDA can contradict this situation. One may prefer to use a lower determination limit to provide more sensitivity at the expense of more false positives, and GRABGAM effectively provides this option within its " $3 \sigma / x \sigma$ " formalism for flagged MDA peaks. Other approaches to detection limits have also been offered, such as the use of direct Poisson statistics [28] and individual peak channel evaluations for peak areas [14].

\subsection{Algorithm Features and Limitations}

The peak search algorithm uses filters that trap the area above displaced adjacent background regions. GRABGAM was originally developed for $2000 \mathrm{keV}$ spectra spanning 4000 channels; thus, a filter with a $4.0 \mathrm{keV}$ ( 8 channels) gross region was selected to contain essentially all of the area of a $1332 \mathrm{keV}$ gamma peak with FWHM of $2.0 \mathrm{keV}$. Adjacent background regions of $2.0 \mathrm{keV}$ (4 channels) displaced from the peak region by $2.0 \mathrm{keV}$ ( 4 channels) then effected a good signal-to-background selection, even for a significantly larger FWHM. For the multiplet analysis, similar smaller filters with gross regions of $1.0 \mathrm{keV}$ (2 channels) and $2.0 \mathrm{keV}$ ( 4 channels) were used to help resolve peaks. These filters also have the potential to accentuate the signal to background; however, the $1.0 \mathrm{keV}$ filter could miss evidence of a peak indicated by two high count channels relative to two directly adjacent channels, depending on the counts in the displaced background channels. On the other hand, such behavior may also be consistent with statistical fluctuations, so that such a missed "detection" by GRABGAM is not considered a serious loss. Overall, the GRABGAM filters find peaks that can be comparable to or somewhat broader than those of the user input FWHM* for the analysis, and the detailed printout reveals whether the peak is a true singlet or a broader composite.

The singlet algorithms do not depend on fitting parameters for a particular model, which is customary for differential peak fitting [2-3]. Instead, the adjacent background ROIs are selected by searching for the flat regions on either side of the peak. Thus, even if the peak is a pure Gaussian, smeared by amplifier drift, or degraded by a tail, the calculated peak area is the full-energy gamma response within the gross peak region; additionally, the integral $F$ analysis provides adequate values for median, FWHM, and tail. A peak found by the search algorithm will be rejected if the subsequent peak analysis indicates $A<3 \sigma$; this possibility exists since the signal-to-noise enhancement of the filters may cause peak 
identification for statistical fluctuations. However, experience has shown that the combination of search and singlet algorithms effectively eliminate these false positive cases. Also, GRABGAM has not exhibited detection of other spectral artifices such as Compton edges or backscatter.

For singlets, the $\mathrm{F}$ analysis assumes that constant adjacent background regions exist, which is generally true in low-level gamma spectrometry, as such background regions are normally determined within statistical requirements. However, a significantly variable background does impact an $F$ analysis which assumes $v(E)=0$, as illustrated in Fig. 13. In this example, the $F$ analysis is in error because the average background on the lowerenergy side of the peak is higher than nearby points of the peak region and the average background on the higher-energy side is lower. Thus, the $F$ values will be negative on the low-energy side of the peak and also modified on the high-energy side. Yet, the singlet area is essentially unaffected, and the centroid, FWHM, and tail experience only minor inconsequential impacts.

For multiplets, the $\mathbf{F}$ analysis does model any statistically significant variation $V(E)$ of the background, as the impact can be important. The multiplet algorithms produce an analysis like that of Fig. 4. The F function approach utilizes an individual background subtraction for each gross channel count within the multiplet. The total area $A_{M}$ of all peaks in the multiplet is generally determined with accuracy comparable to that of a singlet peak; however, a calculational error exists for partitioning $A_{M}$ among the individual peaks of the multiplet, as the area $A_{m}$ of an individual peak is approximated as the area between its adjacent minima. The adequacy of the approximation can be tested with the PEAKPART code, which corrects for the ROI contributions from the other peaks of the multiplet.

Further improvement on the current multiplet analysis will require more detailed information on the peak shapes. Ideally, peak shapes could be extracted using the larger peaks of the sample spectrum, so that the intrinsic generality of the GRABGAM analysis for a spectrum is preserved; however, the prospect of a sufficient set of peaks is not likely for low-level samples. Thus, spectral shapes from calibration standards and/or modeling will be required for each detector as well as geometry in some cases, so that the intrinsic generality of the analysis would be compromised. A standard addition method in which identical spiked and unspiked samples are counted would essentially retain intrinsic generality and provide the peak shapes; however, quality spiking of samples with gamma standards can be expensive and labor intensive.

Multiplets with low-level counting statistics can cause additional concerns, and if $F$ is not well-behaved, warning flags are printed with the output. This approach follows the overall GRABGAM philosophy of presenting the user with the option of reevaluating marginal cases rather than automatically removing them from consideration. In practice, with these guidelines and those mentioned above, the GRABGAM multiplet analysis has generally provided a useful tool for appraising closely spaced peaks. 


\subsection{Intrinsic Quality Guides}

The output hardcopies of GRABGAM have provided useful guides for documenting the quality of sample analyses. The initial input summary sheet lists information on all user inputs as a quality check. The peak listing prints both the peak energy determined by the analysis and the energy matched by the isotope library, guiding the user on any need for reanalysis with a refined energy calibration. Also the analysis-determined FWHM and the user-input FWHM* are printed, allowing a quality check on detector performance and advising the user on whether the input FWHM* is adequate in selecting ROI-dependent analyses such as flagged MDAs. Examination of FWHM and tail provide either singlet quality information or flags concerning MDAs, ROI analyses, and multiplet features.

The GRABGAM printouts have also provided consistency checks for the adequacy of detector backgrounds and efficiencies. The spectral peak count rates are printed adjacent to those from background spectra, for direct consistency checks. GRABISOS calculations frequently provide the user with consistency checks on the stability of the detector efficiency curve by comparing the different peak activities deduced for an individual isotope. For example, soil samples often contain radium daughters $B-214$ and $\mathrm{Pb}-214$ that are in equilibrium, whereby several nicely spaced gammas are detected over a range of $200-1800 \mathrm{keV}$; agreement for the isotope activities for these peaks demonstrates the adequacy of the relative efficiencies; this information, coupled with monthly check source tests, affirms the absolute efficiency calibration as well. In summary, the above routine information from GRABGAM analyses provides the user with excellent guidelines for affirming the quality of the results.

\subsection{Performance History}

The GRABGAM code has successfully supported low-level HPGe gamma spectrometry at SRTC for over 14 years, involving analyses of over 10,000 samples. Various projects supported during this period include studies of Chernobyl fallout [24-25], appraisal of LDEF satellite samples for NASA [29], monitoring of radioactive levels in the Savannah River [30-31], evaluation of sediment levels in SRS cooling ponds [31-34], environmental analyses of high volume air samples near. SRS [35], and studies of the Arctic Ocean. [36]. In addition to these, numerous customer service samples have been conducted for SRS and offsite sponsors.

GRABGAM analyses have been applied to both laboratory and field detectors. Laboratory applications include thirteen HPGe detectors with efficiency range of 10-160\%, which have been used in the Ultra-Low-Level counting Facility [21-22] and the Underground Counting Facility $[18,23]$. Two HPGe detectors on the SRS TRAC mobile laboratory [37] also used the GRABGAM analysis for a number of years. One early TRAC applications was the collection and analysis of air samples following the Chernobyl accident, where the TRAC vehicle analyzed regional samples during transits within Georgia and South Carolina [25]. Although microphonics associated 
with the vehicle motion degraded the quality of the spectral peaks, the general analysis with the F-function properly calculated these peaks, giving good agreement for similar samples analyzed at the Ultra-Low-Level Counting Facility [24]. On-location spectra obtained with a HPGe sonde [38] and an HPGe underwater detector [31-34] were also successfully analyzed.

\section{Conclusions}

GRABGAM has performed well in its role for analyzing low-level environmental samples by HPGe analysis. Overall, the analysis has attempted to provide user options for evaluating the presence of marginal but potentially real peaks, while automatically processing peaks with $1-\sigma$ detection statistics that are better than 338 . Evaluation of marginal peaks is possible by conversion of the GRABGAM-formatted MDA data and/or by direct inspection of the spectrum; however, the best option is to continue counting for a time based on the statistics of the marginal peak. None of these options would be available if GRABGAM discarded the marginal peaks.

The general nature of the GRABGAM peak search and analysis does not depend on specific peak shape parameters. Accordingly, other types of spectroscopy are expected to be amenable to the GRABGAM algorithms. Indeed, a NaI version GRABNAI was developed for an underwater NaI detector that has monitored the Savannah River effluent activities from the Savannah River Site and the Plant Vogtle power reactors [30]. While not an advanced NaI peak stripping code, GRABNAI has provided useful gamma peak analyses for time profiles of the effluent discharges, which have correlated well with recorded discharges at the source. Another spinoff is GRABTRIT, which was developed to analyze SRS low-level tritium samples counted within gas proportional counters [21-22]. In this case a spectrum results for the detector signals following their pulse-shape discrimination, which separates cosmic-ray background components from a narrow tritium peak. A code GRABALFA for surface barrier alpha spectrometry has also been written, as features of alpha tailing due to sample thickness should be handled well by the singlet algorithm; however, an algorithm for handling alpha multiplets still needs to be developed. Finally, the peak search algorithm was utilized in a gas chromatography spectral analysis code at SRS.

\section{Acknowledgements}

The author gratefully acknowledges those who assisted with GRABGAM development and checkout. Ray Sigg, Martti Kantelo, and Gordon Lien provided input for the initial development, including computer coding techniques, low-level spectroscopy goals, and hardware configuration. Also acting as users, they have been followed by Worth Dalton, C.D. Ouzts, Tim Wilson, and Jim Cadieux, all of whom have provided useful feedback for enhancing GRABGAM over the years. 
[1] W.G. Winn, GRABGAM Analysis of Ultra-Low-Level HPGe Gamma Spectra, US DOE Savannah River Report WSRC-TR-99-00187 (1999).

[2] K. Delbertin and R.G. Helmer, Gamma- and X-Ray Spectrometry with Semiconductor Detectors, North-Holland / Elsevier Science Publishing Co., New York (1988).

[3] C.G. Sanderson, Environmental International 14, 379 (1988).

[4] H.Mancher, Nuclear Instruments and Methods A258, 246 (1987).

[5] A.E. Proctor, EG\&G Idaho Report, 311pp (1987).

[6] W.K. Hensley, E.A. Lepel, M.E. Yuly, K.H. Abel, Journal of Radioanalytical and Nuclear Chemistry 124, 481 (1988).

[7] R.B. Welch, F. Gyger, D.T. Jost, H.R. Von Gunton, U. Krahenbuhl, Nuclear Instruments and Methods A269, 615 (1988).

[8] E.W. Killian and J.K. Hartwell, IEEE Transactions in Nuclear Science 36, 615 (1989).

[9] J.S. Yadav, J. Bruckner, J.R. Arnold, Nuclear Instruments and Methods A277, 591 (1989).

[10] V. Hnatowicz, Computer Physics Communications Netherlands 60,111 (1990).

[11] T.J. Kennett, W.V. Prestwich, Nuclear Instruments and Methods A297, 315 (1990).

[12] L.C. Longoria, A.H. Naboulsi, P.W. Gray, T.D. MacMahon, Nuclear Instruments and Methods A299, 308 (1990).

[13] L.A. Hammond, P.W. Gray, A.H. Naboulsi, T.D. MacMahon, Nuclear Instruments and Methods A334, 543 (1993).

[14] T.P. Luedeke, G.E. Tripard, Nuclear Instruments and Methods A372, 283 (1996).

[15] C.V. Hampton, B. Lian, Wm.C. McHarris, Nuclear Instruments and Methods A353, 280 (1994).

[16] I. Meray, Nuclear Instruments and Methods A353, 272 (1994).

[17] N.D. Gagunashvili, Nuclear Instruments and Methods A343, 606 (1994).

[18] W.G. Winn, Trans. Am. Nuc. Soc. 54, 34 (1987).

[19] L.A. Currie, Analytical Chemistry 40, 586 (1968). 
[20] W.G. Winn, Nuclear Technology, 103, 162 (1993).

[21] W.W. Bowman, M.B. Hughes, Methods of Low-Level Counting and Spectrometry (IAEA Proceedings series, Berlin, April 6-7, $1981), 353(1981)$.

[22] R.C. Hochel, D.W. Hayes, Nuclear Instruments and Methods 130,183 (1975).

[23] W.G. Winn, W.W. Bowman, A.L. Boni, The Science of the Total Environment 69, 107 (1988).

[24] W.G. Winn and M.V. Kantelo, Trans. Am. Nuc. Soc, 60, 75 (1989)

[25] R.A. Sigg, Trans. Am. Nuc. Soc. 60, 73 (1989).

[26] M.A. Mariscotti, Nuclear Instruments and Methods 50, 309 (1967).

[27] N. Markevich, I. Gertner, Nuclear Instruments and Methods A283, 72 (1989).

[28] G. Bernasconi, E.D. Greaves, I. Sajo-Bohus, Nuclear Instruments and Methods A307, 491 (1991).

[29] W.G. Winn, NASA Conference Publication 3134 (Kissimee, FL), Part 1, 287 (1991).

[30] W.G. Winn, S. Sundaram, ANS Topical Meeting Proceedings ISBN : 0-89448-188-6 (Charleston, SC), 101 (1993).

[31] W.G. Winn, Journal of Radioanalytical and Nuclear Chemistry 194, no 2, 334 (1995).

[32] W.G. Winn, Trans. Am. Nuc. Soc. 66, 32 (1992).

[33] W.G. Winn, D.L. Dunn, P.J. Bresnahan, Trans. Am. Nuc. Soc. 75, $54(1996)$.

[34] D.I. Dunn, W.G. Winn, P.J. Bresnahan, Trans. Am. Nuc. Soc. 75, 52 (1996).

[35] W.G. Winn, J.R. Cadieux, Trans. Am. Nuc. Soc., 486 (1997).

[36] E.R. Landa, E. Reimnitz, D.M. Beals, J.M. Pochowski, W.G. Winn, I. Rigor, Arctic, 27 (1998).

[37] R.A. Sigg, ANS Topical Meeting Proceedings, CONF-860932 (Bethesda, MD), 131 (1986).

[38] W.G. Winn, K.J. Hofstetter, K.W. MacMurdo, Trans. Am. Nuc. Soc. 73,83 (1995). 


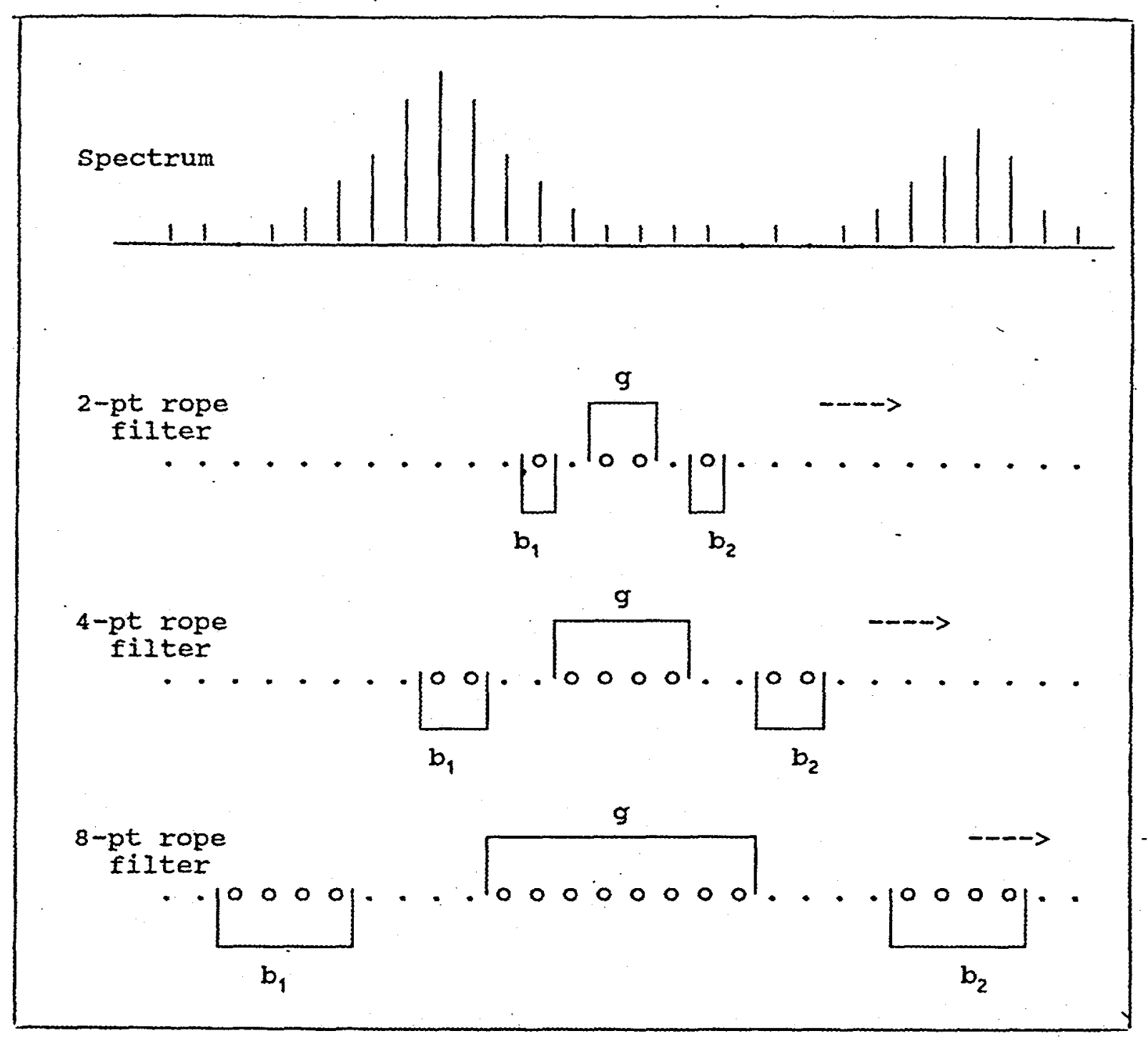

Fig. 1. Spectrum examined by three different size filters. Each filter sums spectral counts $g$ in the middle channels and counts $b_{1}$ and $b_{2}$ in adjacent channels. Each filter traps its own counts $a_{f}=g-b_{1}-b_{2}$ with counting $\sigma=\left(g+b_{1}+b_{2}\right){ }^{1 / 2}$. If $a_{f} \geq 3 \sigma$, a candidate peak is retained for further analysis. 

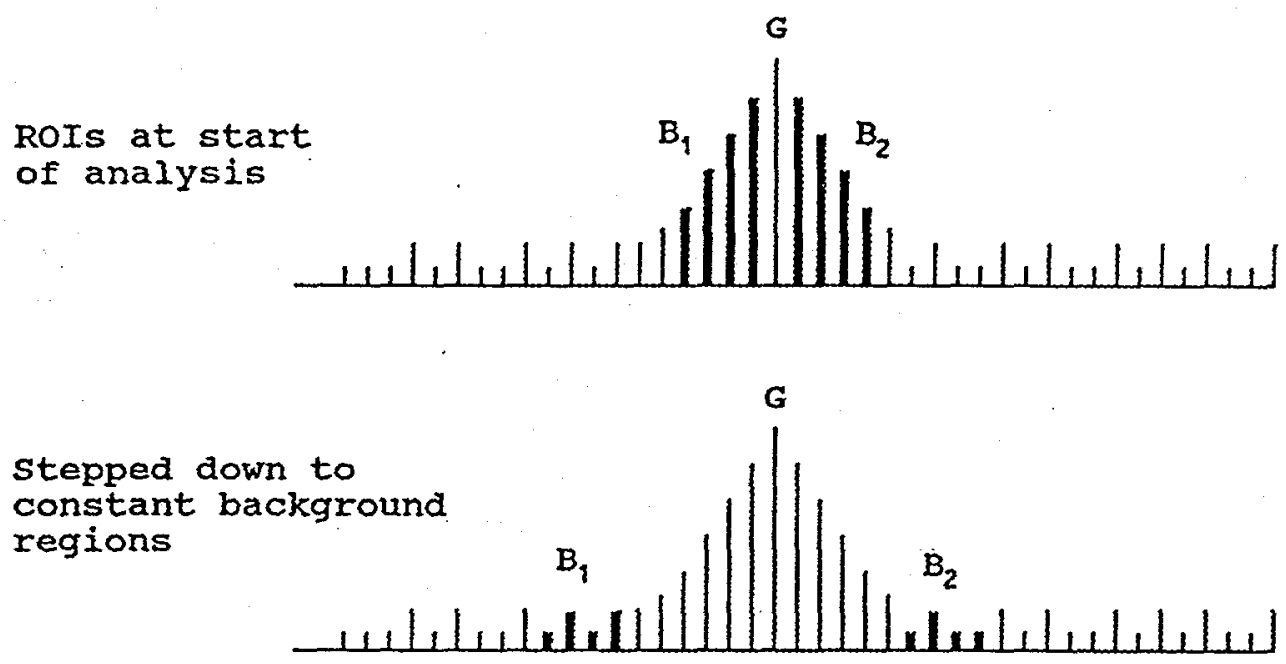

Adjusted for even number of gross channels

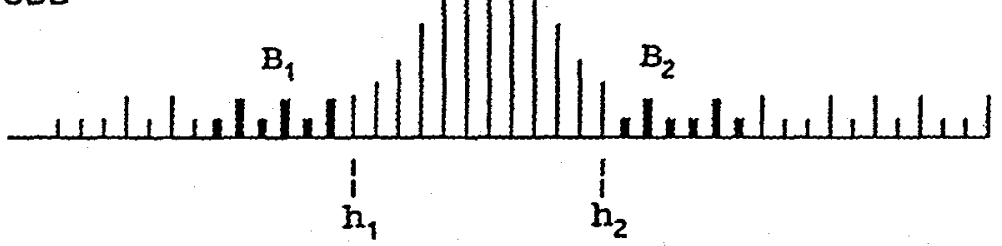

Fig. 2. Singlet analysis for RoIs of gross (G) and background $\left(\mathrm{B}_{1}, \mathrm{~B}_{2}\right)$ counts. The two background RoIs are found such their corresponding adjacent gross channels $\left(h_{1}\right.$ and $h_{2}$ ) have counts that do not exceed their respective background channel averages by more than $2 \sigma$. 
(a) perfect resolution

A $\delta\left(E-E_{g}\right)$

full energy

peak

b $\left[1-\mathrm{H}\left(E-E_{\mathrm{g}}\right)\right]$

step background

$\delta\left(E-E_{g}\right)=d H\left(E-E_{g}\right) / d E$

$\mathrm{b}$

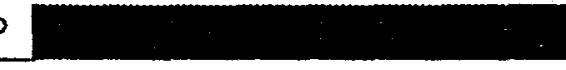

$\mathbf{E}_{\mathrm{g}}$

$E$

(b) actual resolution

b $\left[1-F\left(E-E_{g}\right)\right]$

step background

$A f\left(E-E_{g}\right)$

full energy

peak

b

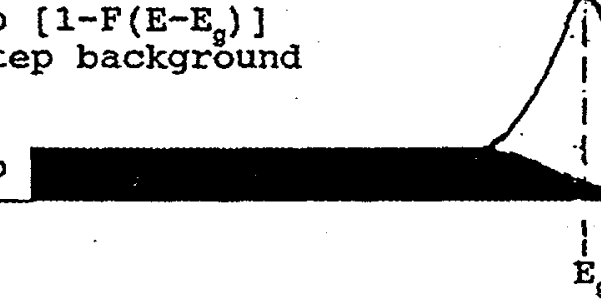

$$
f\left(E-E_{g}\right)=d F\left(E-E_{g}\right) / d E
$$

(c) flagged analyses (flag = negative tail)

Flag

$-1$

$-2$

$-3$

$-4$

$-5$
Meaning

MDA forced/no peak

$$
F<0
$$

$\mathrm{F} \longleftrightarrow 0.16,0.50,0.84$

SigB-SigA $=0$

sigB-Cent $=0$
Response

None - MDA analysis FWHM big, Cent est FWHM big, Cent est overridden by $-2,3$ overridden by $-2,3$

Fig. 3. Detected gamma response function for (a) perfect solution and (b) actual solution, along with (c) flags indicating nature of analysis. It can be shown that convoluting $\delta\left(E^{\prime}-E_{g}\right)$ and $H\left(E^{\prime}-E_{g}\right)$ with $f(E-$ $\left.E^{\prime}\right)$ Yields the $f\left(E-E_{g}\right)$ and $F\left(E-E_{g}\right)$ above. 
(a) Multiplet area

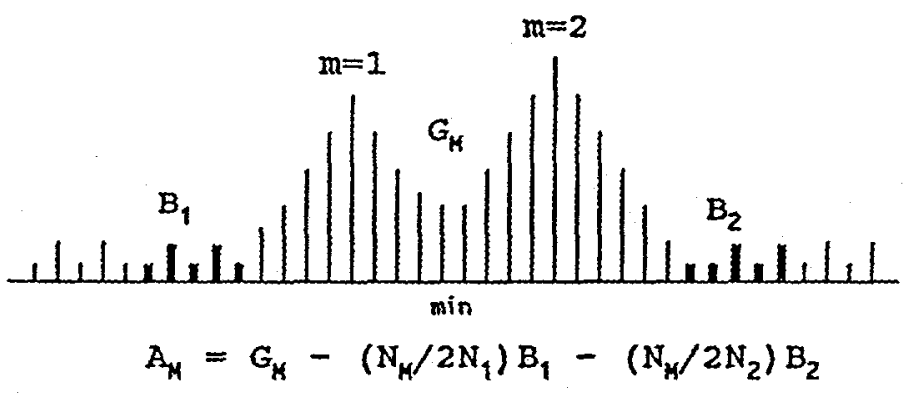

(b) Peak areas

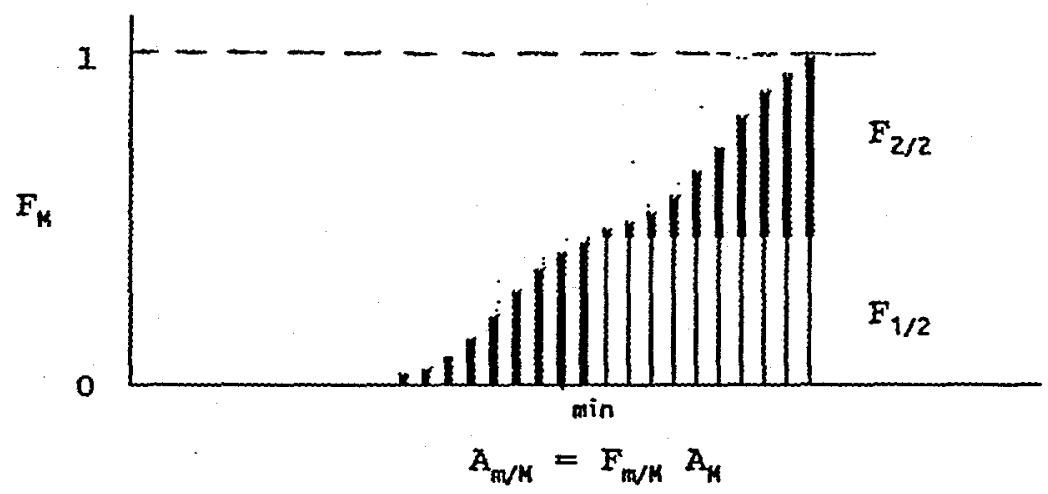

(c) Flagged analyses

$$
\begin{aligned}
& (F W H M=M+F l a g A / 10+F l a g B / 100) \\
& (T a i 1=m+F l a g A / 10+F l a g B / 100)
\end{aligned}
$$

Problem FlagA FlagB Response

$\begin{array}{lcll}F_{m / M} \leq 0 & 1 & 0 & \text { Use linear background } B_{L} \\ B_{L}<0 & 1 & 1 & A_{m / M} \text { with warning } \\ A_{m / M}<0 & 2 & 1 & \text { Backgrounds adjacent minima } \\ A_{M} \leq 3 \sigma & 0,1,2 & 2 & \text { Use lower } B_{1}, B_{2} \text { or fix } A<0\end{array}$

Fig. 4. Multiplet analysis: (a) Calculation of total multiplet area $A_{4}$, similar to that for singlet; (b) individual peak areas $A_{m / M}$ calculated from $A_{M}$ and $F_{m / M}$; (c) flagged multiplet analyses for reconsideration. 


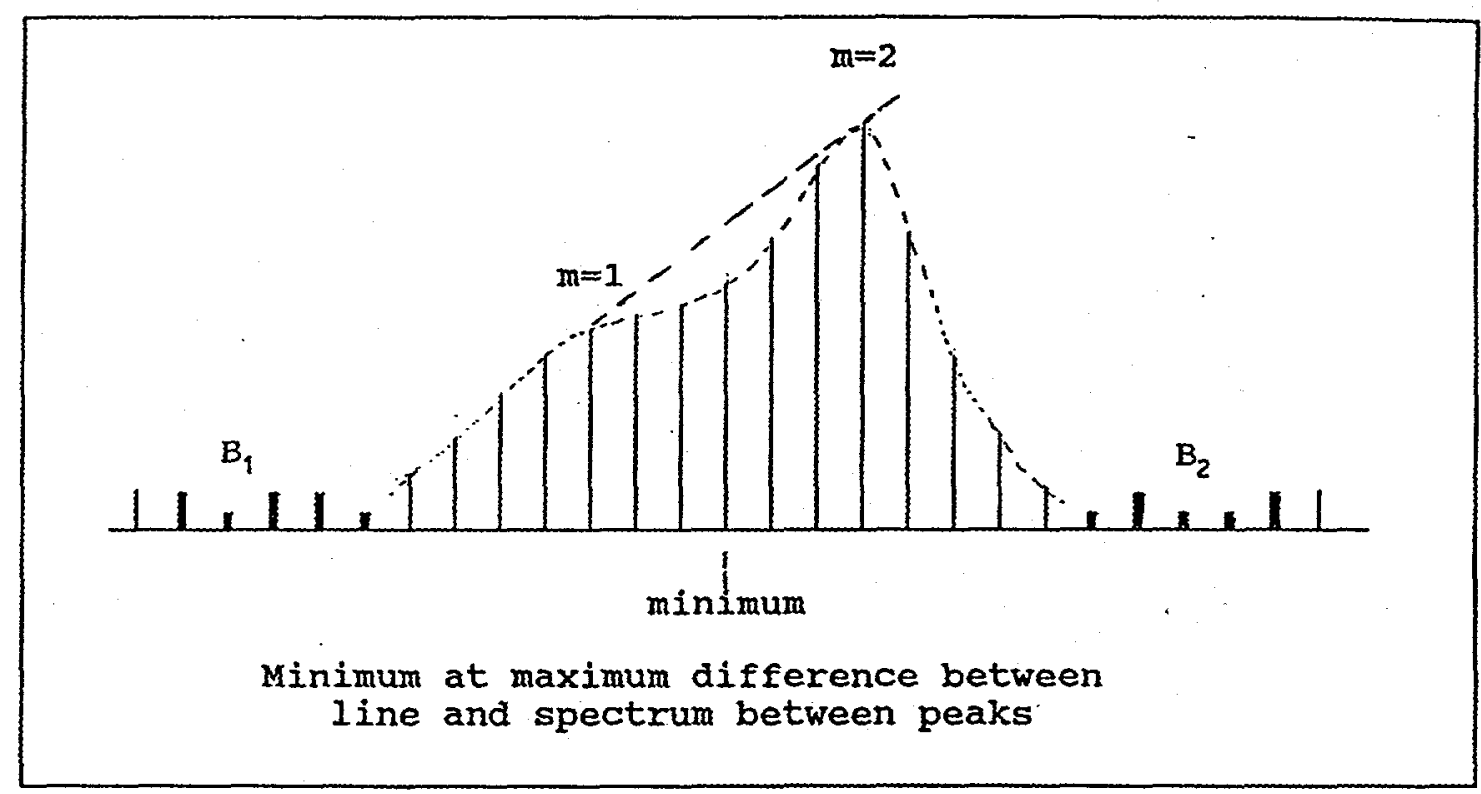

Fig. 5. Multiplet minimum for "shoulder doublet". 


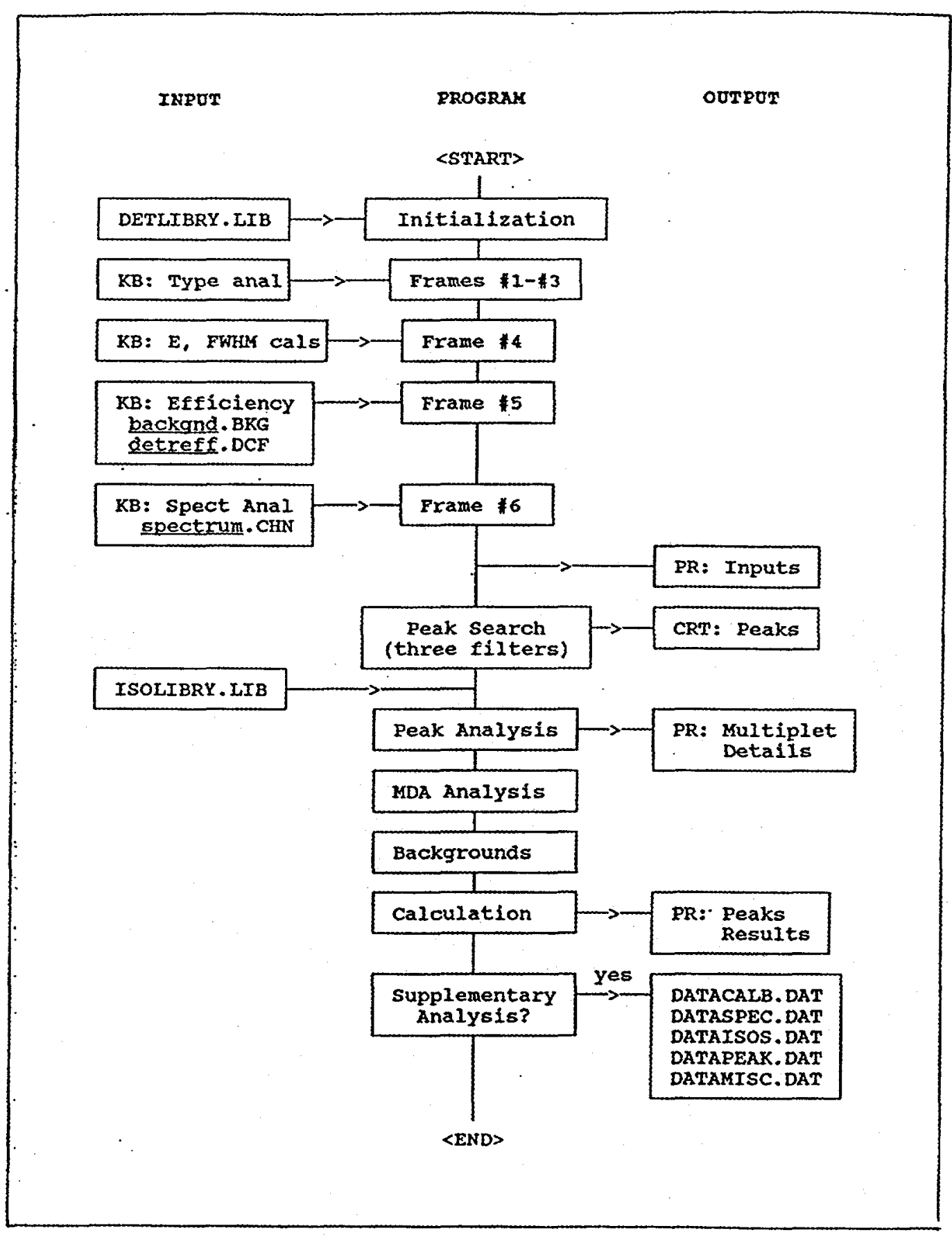

Fig. 6. GRABGAM program sequence relative to input and output of keyboard (KB), printer (PR), video monitor (CRT) and disk files $(x \times x \times x . x \times x)$. 


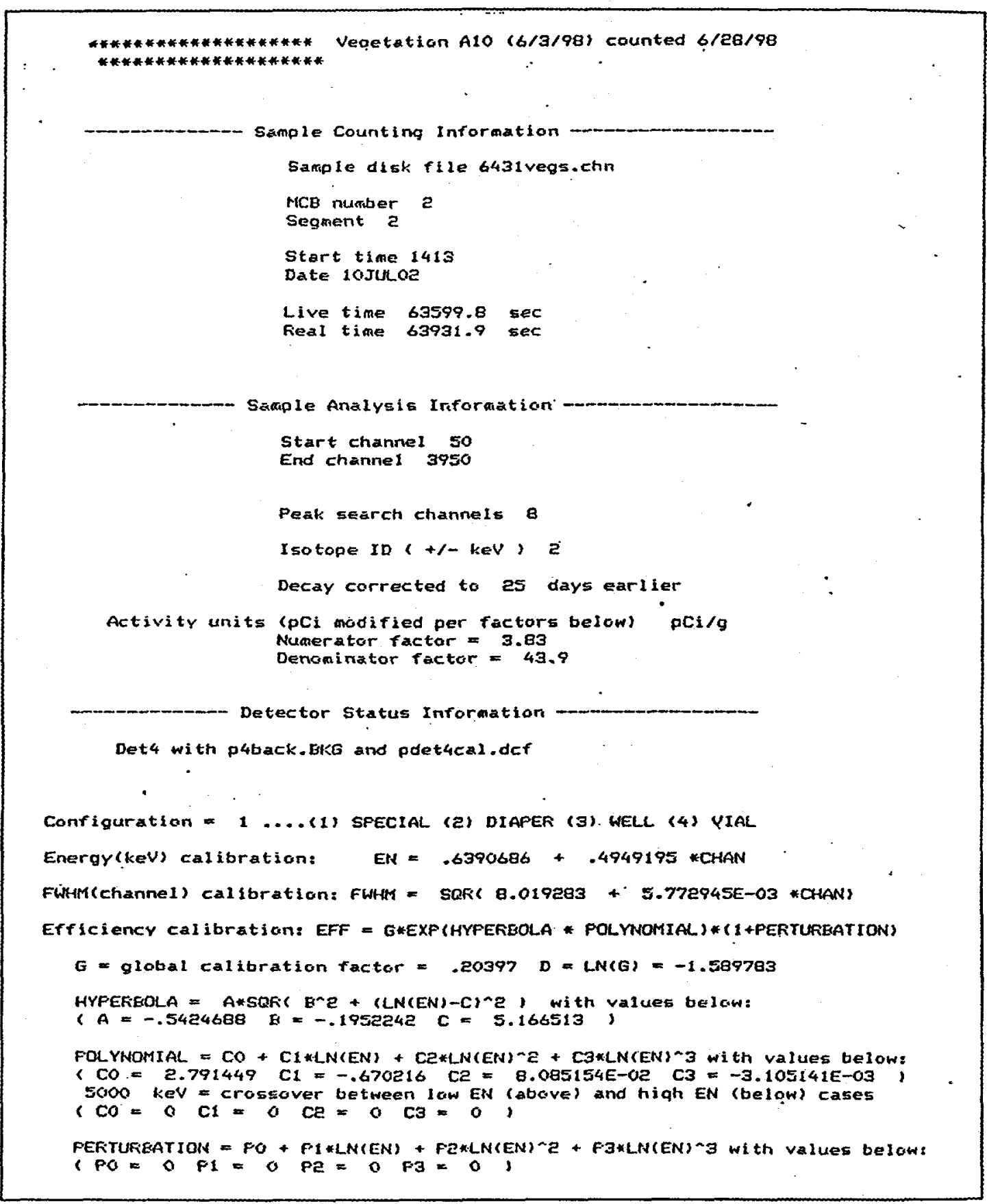

Fig. 7. printout of input parameters 


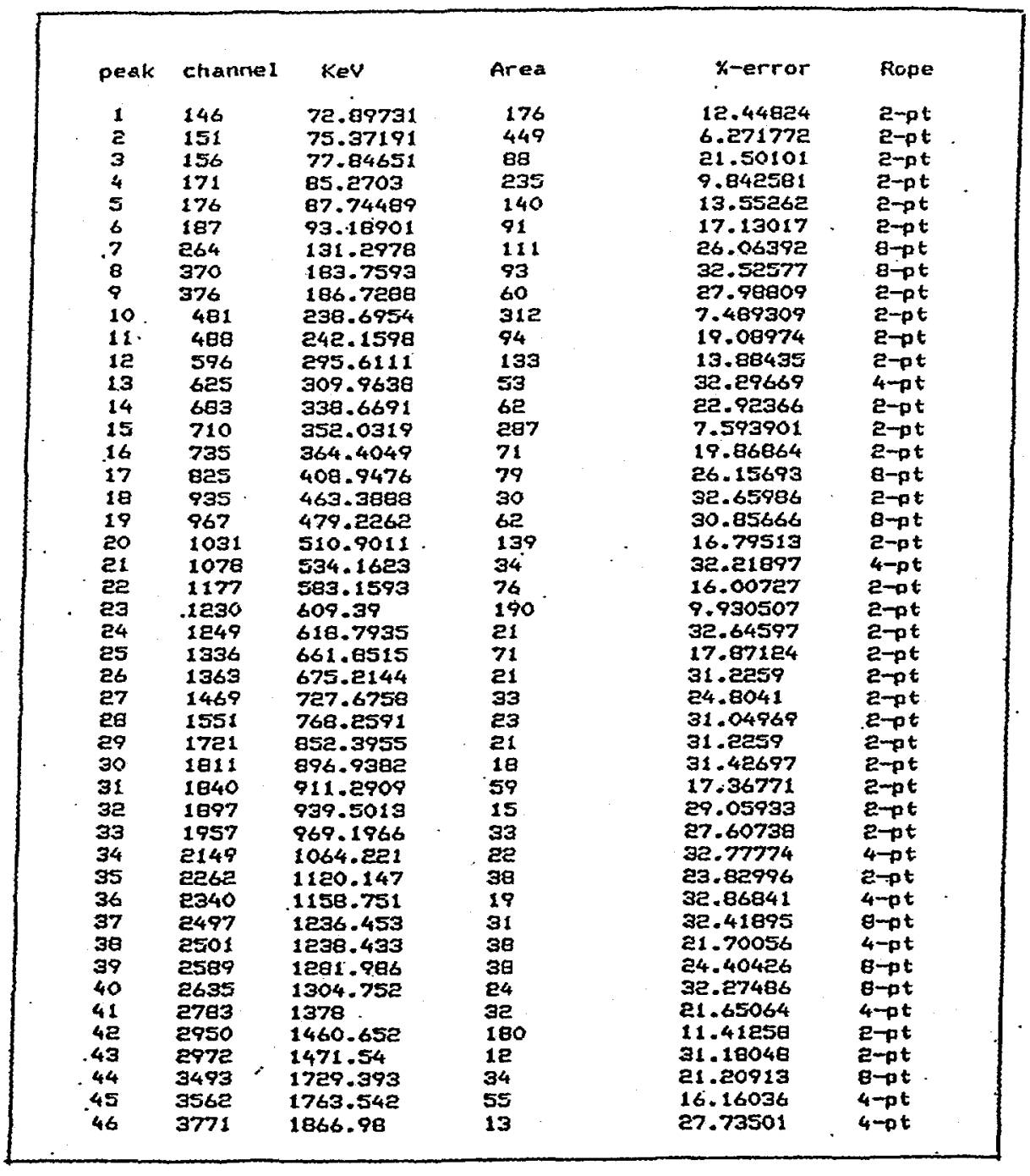

Fig. 8. Example of candidate peaks table displayed on monitor. 


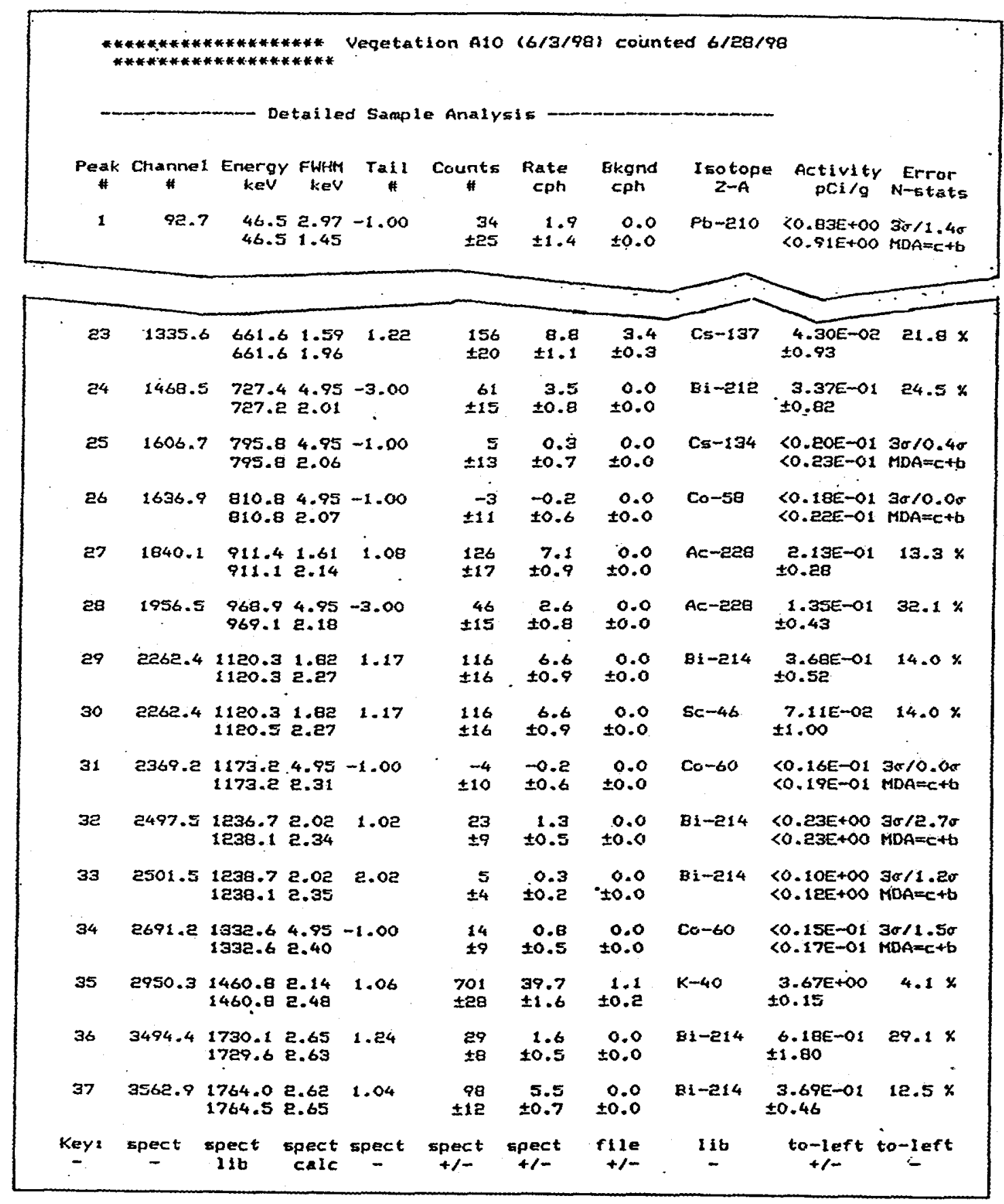

Fig. 9. Peak analysis printout table 
Multiplet Peak Details

\begin{tabular}{|c|c|c|c|c|c|c|c|c|}
\hline $\begin{array}{l}\text { Feak } \\
\text { \# }\end{array}$ & mini & $\begin{array}{l}\text { Channel } \\
\max \end{array}$ & mina & $\begin{array}{l}\text { Area } \\
\text { counts }\end{array}$ & $\begin{array}{l}+1- \\
+1-\end{array}$ & $\begin{array}{l}\text { Errar } \\
\text { caunts }\end{array}$ & $\underset{k \in V}{\text { Energy }}$ & $\begin{array}{c}\text { Isatcape } \\
Z-A\end{array}$ \\
\hline 1 & $\begin{array}{l}147.5 \\
154.5\end{array}$ & $\begin{array}{l}152.5 \\
157.4\end{array}$ & $\begin{array}{l}154.51 \\
161.50\end{array}$ & $\begin{array}{l}68 \\
37\end{array}$ & $\begin{array}{l}+1- \\
+1-\end{array}$ & $\begin{array}{l}26 \\
19\end{array}$ & $\begin{array}{l}75.1 \\
77.6\end{array}$ & $\begin{array}{l}\text { FoEIKX } \\
\text { Wo-Lib }\end{array}$ \\
\hline $\begin{array}{l}1 \\
2\end{array}$ & $\begin{array}{l}468.5 \\
476.5\end{array}$ & $\begin{array}{l}473.4 \\
479.5\end{array}$ & $\begin{array}{l}476.51 \\
483.50\end{array}$ & $\begin{array}{r}109 \\
50\end{array}$ & $\begin{array}{l}+1- \\
+1-\end{array}$ & $\begin{array}{l}32 \\
21\end{array}$ & $\begin{array}{l}239.0 \\
242.0\end{array}$ & $\begin{array}{l}\text { Fb-212 } \\
\text { Fb-214. }\end{array}$ \\
\hline $\begin{array}{l}1 \\
2\end{array}$ & $\begin{array}{l}1112.5 \\
1119.5\end{array}$ & $\begin{array}{l}1118.8 \\
1122.5\end{array}$ & $\begin{array}{l}1119.53 \\
1127.50\end{array}$ & 39 & $\begin{array}{l}+1- \\
+1-\end{array}$ & $\begin{array}{r}4 \\
13\end{array}$ & $\begin{array}{l}568.4 \\
570.3\end{array}$ & $\begin{array}{l}C s-134 \\
C s-134\end{array}$ \\
\hline $\begin{array}{l}1 \\
2\end{array}$ & $\begin{array}{l}1422.5 \\
1429.5\end{array}$ & $\begin{array}{l}1428.7 \\
1430.3\end{array}$ & $\begin{array}{l}1429.53 \\
1435.50\end{array}$ & $\begin{array}{l}10 \\
16\end{array}$ & $\begin{array}{l}+1- \\
+1-\end{array}$ & $\begin{array}{l}5 \\
6\end{array}$ & $\begin{array}{l}726.7 \\
727.5\end{array}$ & $\begin{array}{l}\mathrm{Bi}-21 \mathrm{e} \\
\mathrm{Ei}-21 \mathrm{E}\end{array}$ \\
\hline $\begin{array}{l}1 \\
2 \\
3\end{array}$ & $\begin{array}{l}1570.5 \\
1578.5 \\
1580.5\end{array}$ & $\begin{array}{l}1576.9 \\
1579.5 \\
1581.5\end{array}$ & $\begin{array}{l}1578.53 \\
1580.53 \\
1586.50\end{array}$ & $\begin{array}{r}10 \\
6 \\
7\end{array}$ & $\begin{array}{l}+1- \\
+1- \\
+1-\end{array}$ & $\begin{array}{l}6 \\
5 \\
5\end{array}$ & $\begin{array}{l}802.4 \\
803.7 \\
804.7\end{array}$ & $\begin{array}{l}C s-134 \\
C s-134 \\
B i-214\end{array}$ \\
\hline $\begin{array}{l}1 \\
2\end{array}$ & $\begin{array}{l}2695.5 \\
2703.5\end{array}$ & $\begin{array}{l}2702.7 \\
2706.6\end{array}$ & $\begin{array}{l}2703.53 \\
2712.50\end{array}$ & $\begin{array}{r}7 \\
17\end{array}$ & $\begin{array}{l}+1- \\
+1-\end{array}$ & $\begin{array}{l}3 \\
5\end{array}$ & $\begin{array}{l}1377.2 \\
1379.2\end{array}$ & $\begin{array}{l}B i-214 \\
B i-214\end{array}$ \\
\hline
\end{tabular}

Fig. 10. GRABGAM multiplet printout. Note that min2 format $[\mathrm{XxXx} \cdot \mathrm{XY}]$ provides channel number as $[\mathrm{XxXx} \cdot \mathrm{x}]$, while [Y] equals [0] for end minimum associated with $h_{2}$, [1] for absolute minimum, [2] for relative minimum, and [3] inverse center of gravity minimum. 


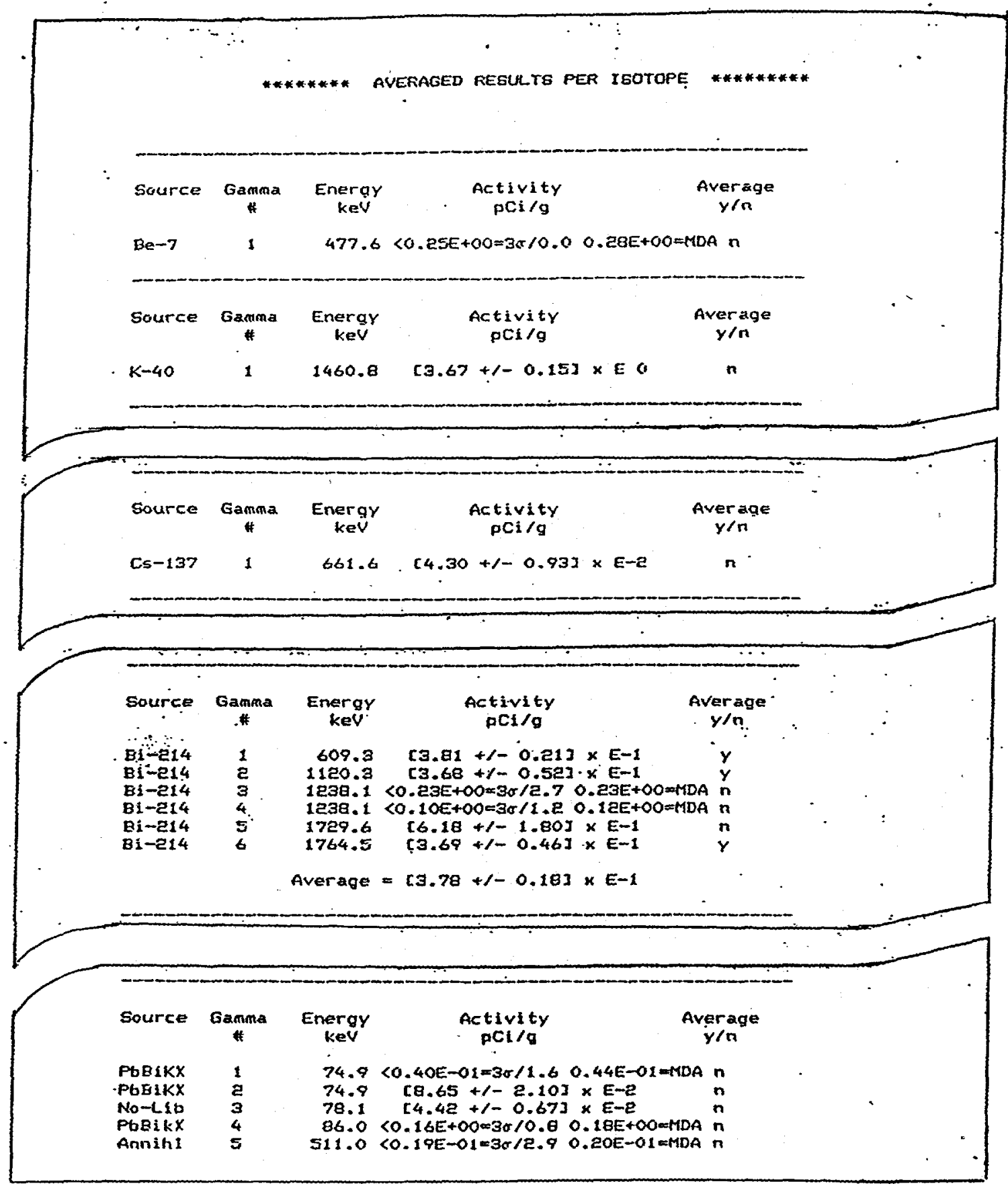

Fig. II. GRABISOS isotope printout 


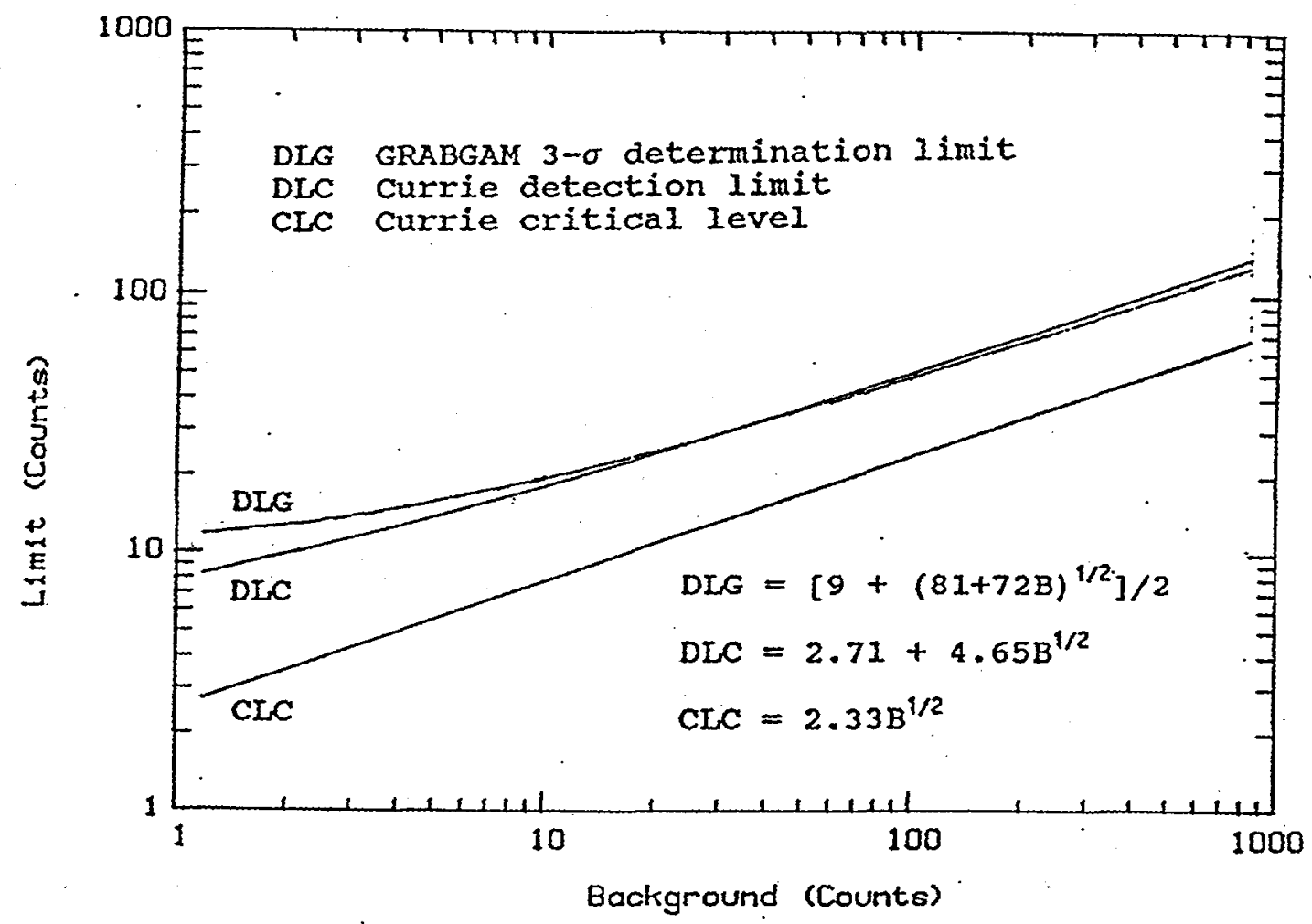

Fig. 12. Comparison of detection level scenarios as a function of background $\mathrm{B}$. 

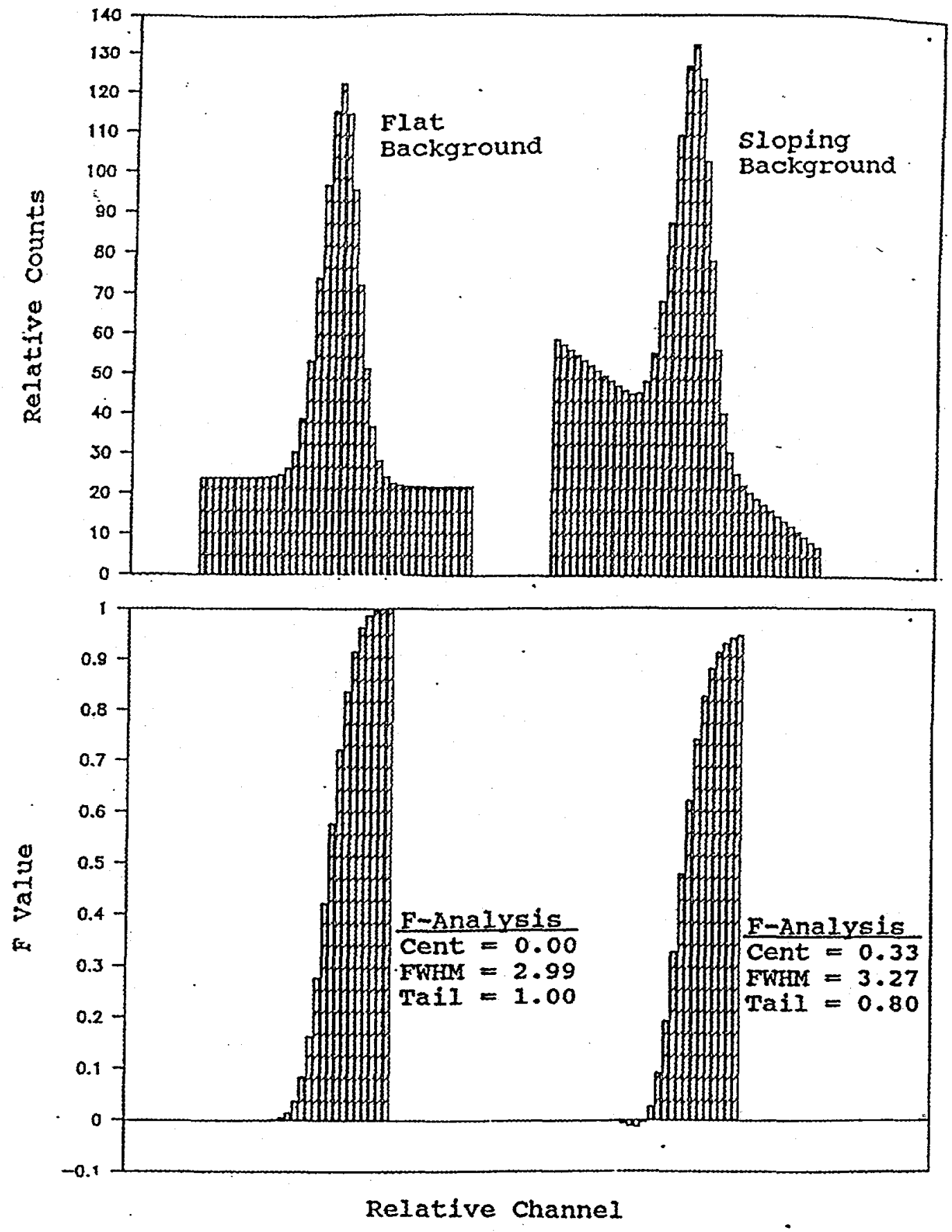

Fig. 13. Effect of sloping background on F-analysis assuming that $v(E)=0$, by comparing Gaussian peak (centroid $\equiv 0.00$, FWHM $\equiv 3.00$, tail $\equiv 1.00$ ) on flat and sloping backgrounds. 